\title{
Superdense Coding of Quantum States
}

\author{
Aram Harrow \\ Physics Department, MIT, 77 Massachusetts Avenue, Cambridge, Massachusetts 02139, USA \\ Patrick Hayden and Debbie Leung \\ Institute for Quantum Information, Caltech 107-81, Pasadena, California 91125, USA
}

(Received 30 August 2003; published 4 May 2004)

\begin{abstract}
We describe a method for nonobliviously communicating a $2 l$-qubit quantum state by physically transmitting $l+o(l)$ qubits, and by consuming $l$ ebits of entanglement plus some shared random bits. In the nonoblivious scenario, the sender has a classical description of the state to be communicated. Our method can be used to communicate states that are pure or entangled with the sender's system; $l+o(l)$ and $3 l+o(l)$ shared random bits are sufficient, respectively.
\end{abstract}

DOI: $10.1103 /$ PhysRevLett.92.187901

PACS numbers: 03.67.Hk, 03.65.Ta

Introduction.-One of the most striking effects in quantum information theory is known as superdense coding [1]. By making use of shared entanglement, it is possible to communicate classical information at twice the rate one would naively expect is allowed by causality. That is, by physically transmitting only one qubit (a twolevel system such as a spin- $1 / 2$ particle) while at the same time consuming one ebit [the shared state $(|00\rangle+$ $|11\rangle) / \sqrt{2}]$, it is possible to communicate two classical bits worth of information. This observation can be summarized by the following schematic inequality:

$$
1 \text { qubit }+1 \text { ebit } \succeq 2 \text { cbits. }
$$

It is natural to ask whether it is possible, using the same resources, to communicate two qubits worth of quantum information rather than just two classical bits. A simple thought experiment reveals that this should not be the case. Indeed, if the schematic inequality

$$
1 \text { qubit }+1 \text { ebit } \succeq 2 \text { qubits }
$$

were true, then the two qubits communicated using just a qubit and an ebit could themselves be paired with two ebits, resulting in the communication of four qubits worth of quantum information. Repeating the process, an arbitrary amount of quantum information could be transmitted by sending just the single original qubit and a correspondingly large amount of entanglement. This is known to be impossible [2]. For similar reasons, entanglement cannot increase the quantum capacity of a noiseless quantum channel. This leads to a strong dichotomy: entanglement can double the classical communication capacity of a noiseless quantum channel but does not increase the quantum communication capacity at all.

The argument for the latter claim rests on an important assumption about quantum communication, however, that the sender (Alice) forwards or delivers a quantum state without knowing what it is-a condition known as "oblivious encoding." Equivalently, Alice's action is required to be independent of the transmitted state. Oblivious quantum communication automatically preserves entanglement between the transmitted state and any other system. In fact, recursive use of superdense coding as described in the previous paragraph requires that it preserve entanglement between the transmitted and the receiver's (Bob's) systems.

Thus, the situation can be very different if Alice is given a classical description of the state to be communicated and can alter her encoding operation accordingly; we call such encoding "nonoblivious." An important example of nonoblivious encoding is when Alice chooses the communicated state herself, as is the case, for instance, with quantum digital signatures [3]. Nonoblivious encoding can be more powerful than the oblivious version. This is true, for example, in remote state preparation [4], the variant of teleportation [5] in which the sender knows the state to be communicated. Remote state preparation requires only half of the communication resources used in teleportation [6].

In this Letter we focus on nonoblivious communication of quantum states using the dual resources of quantum communication and entanglement. This is analogous to remote state preparation but the classical communication is now replaced by quantum communication. We restrict our discussion to the tasks of "preparing" a pure state in Bob's system or "sharing" a pure state that is entangled between Alice's and Bob's systems. (We avoid the term "transmitted state," which can be confused with the system that is physically transmitted.) Our goal is to find out the extent to which entanglement can improve the quantum communication capacity of a noiseless quantum channel in the nonoblivious scenario.

Statement of result.-Our main result is that an arbitrary $2 l$-qubit state can be prepared or shared with high probability if Alice transmits $l+o(l)$ qubits to Bob and if they share $l$ ebits and at most $O(l)$ random classical bits. 
No shared randomness is needed in the important cases of communicating tensor product states or arbitrary states drawn according to a known probability distribution. Our result is a generalization of superdense coding to quantum states in the asymptotic and nonoblivious scenario. The possibility of superdense coding hinges on nonobliviousness. By Holevo's theorem [2], both versions of superdense coding use a minimal amount of communication, a rate at which the entanglement cost is also optimal.

In the following, we first discuss the task of preparing pure states, starting with a protocol that requires no shared randomness but only succeeds with some potentially small probability. We then describe a slight modification that uses shared randomness to ensure a high probability of success for all states. Finally, we describe a generalization to share pure entangled states between Alice and Bob.

An exact probabilistic protocol.-We first describe a protocol for Alice to prepare any $d^{2}$-dimensional state $|\psi\rangle$ in Bob's system by sending $\log d$ qubits and consuming $\log d$ ebits of shared entanglement. The protocol succeeds with a probability that depends on the state prepared. Fix a basis $\left\{|i\rangle_{A}|j\rangle_{B}\right\}_{1 \leq i, j \leq d}$ for $\mathbb{C}^{d} \otimes \mathbb{C}^{d}$ (or equivalently $\mathbb{C}^{d^{2}}$ ). Alice and Bob initially share $\log d$ ebits, or equivalently the maximally entangled state $\left|\Phi_{d}\right\rangle=(1 / \sqrt{d}) \sum_{i=1}^{d}|i\rangle_{A}|i\rangle_{B}$. The state to be prepared, $|\psi\rangle$, can always be written as

$$
|\psi\rangle=\frac{1}{\sqrt{d}} \sum_{i, j} x_{i, j}|i\rangle_{A}|j\rangle_{B}=(X \otimes I)\left|\Phi_{d}\right\rangle,
$$

where $X:=\sum_{i, j} x_{i, j}|i\rangle\langle j|$. The identity

$$
\rho_{B}:=\operatorname{Tr}_{A}|\psi\rangle\langle\psi|=\frac{1}{d} X^{T} X^{*}
$$

will be useful later. Equation (1) provides a simple scheme to prepare the state $|\psi\rangle$-Alice applies $X$ to her half of $\left|\Phi_{d}\right\rangle$ and then sends it to Bob:

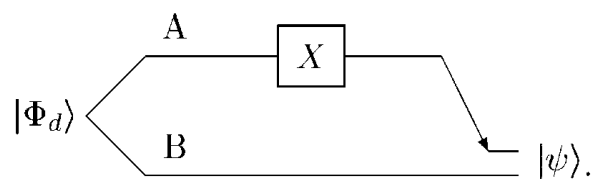

In the above circuit and throughout this Letter, time goes from left to right, single lines represent quantum registers, and registers connected in the left are initially in a maximally entangled state. The arrow in Eq. (3) represents a register sent from Alice to Bob.

This scheme succeeds only if $X$ is applied successfully, which only occurs with some probability, because $X$ may not be unitary. One way to perform $X$ is via a generalized measurement [7] $\rho \rightarrow \sum_{k} E_{k} \rho E_{k}^{\dagger}$ with Kraus operators

$$
E_{0}=\frac{X}{\|X\|_{\infty}}, \quad E_{1}=\sqrt{I-E_{0}^{\dagger} E_{0}},
$$

where the operator norm of $X,\|X\|_{\infty}$, can be taken to be the square root of the largest eigenvalue of $X^{\dagger} X$. Then, $E_{0}^{\dagger} E_{0} \leq I$ and the measurement is well defined. When the measurement outcome is $0, X$ is successfully applied, and this occurs with probability

$$
\operatorname{Tr} E_{0}^{\dagger} E_{0} \frac{I}{d}=\frac{\operatorname{Tr} X^{\dagger} X}{d\left\|X^{\dagger} X\right\|_{\infty}}=\frac{1}{d\left\|\rho_{B}\right\|_{\infty}} \geq \frac{1}{1+\epsilon} .
$$

We have used Eq. (2) to obtain the equalities in Eq. (5). The parameter $\epsilon$ is defined by $\left\|\rho_{B}\right\|_{\infty} \leq \frac{1}{d}(1+\epsilon)$. It measures the deviation of the state $|\psi\rangle$ from being maximally entangled. This probability of success ranges from 1 for a maximally entangled state $(\epsilon=0)$ to $1 / d$ for a product state $(\epsilon=d-1)$. This implementation of $X$ requires the measurement outcome be sent to Bob, but it suffices to use one extra qubit of communication, which is negligible for large $d$.

High probability protocol for arbitrary pure states.If $|\psi\rangle$ is chosen randomly, then with high probability it will be highly entangled ( $\epsilon$ is small) [8-10] and thus we would expect the protocol described above to succeed with high probability.

Suppose our goal is to find a protocol that succeeds with high probability for any choice of input state $|\psi\rangle$, including product states. The above protocol can be adapted easily if Alice and Bob share correlated random bits; Alice will instead prepare $U|\psi\rangle$, with $U$ chosen according to random bits shared with Bob. Bob can then undo $U$ after receiving $U|\psi\rangle$. With high probability, the totally random $U|\psi\rangle$ is highly entangled so that the probabilistic protocol would succeed with high probability.

To make this intuition precise, we will analyze how much randomness is required to ensure a given probability of success. The answer is provided by a lemma that is proved in the Appendix.

Lemma 1: Let $0<\epsilon \leq 1$. If $d \geq \frac{10}{\epsilon}$, there exists a set of isometries $\left\{U_{k}\right\}_{k=1}^{n}$, where $n=\frac{120 \ln 2}{\epsilon^{3}} d \log d$, such that

$$
\forall|\psi\rangle, \quad \operatorname{Pr}_{k}\left(\| \operatorname{Tr}_{A} U_{k}|\psi\rangle\langle\psi| U_{k}^{\dagger} \|_{\infty}<\frac{1+\epsilon}{d}\right) \geq 1-\epsilon .
$$

Here, each $U_{k}$ takes $d^{2}$-dimensional states into a Hilbert space $\mathcal{H}_{A} \otimes \mathcal{H}_{B}$, where $\operatorname{dim}\left(\mathcal{H}_{A}\right)=\frac{112 \ln 2}{\epsilon^{2}} d \log d$ and $\operatorname{dim}\left(\mathcal{H}_{B}\right)=d$.

Our lemma states that, for any state $|\psi\rangle$, choosing $U_{k}$ randomly out of $n=\frac{120 \ln 2}{\epsilon^{3}} d \log d$ possibilities will guarantee, with probability at least $1-\epsilon$, that the state $U_{k}|\psi\rangle$ to be prepared has $\left\|\rho_{B}\right\|_{\infty} \leq \frac{1}{d}(1+\epsilon)$ and can be prepared with probability at least $\frac{1}{1+\epsilon}$.

Thus, assuming the setting of Lemma 1, Eq. (6) is a statement that the following protocol will succeed with probability at least $\frac{1-\epsilon}{1+\epsilon}$ for all states $|\psi\rangle$. To send any state $|\psi\rangle$ : (1) Alice and Bob draw a random $k \in\{1, \ldots, n\}$ using $\log n$ bits of shared randomness. Using Eq. (6), the probability that $\| \operatorname{Tr}_{A} U_{k}|\psi\rangle\langle\psi| U_{k}^{\dagger} \|_{\infty}<\frac{1+\epsilon}{d}$ is at least $1-\epsilon$. 
(2) Alice prepares $U_{k}|\psi\rangle$ using Eq. (3) and Bob applies $U_{k}^{\dagger}$ to obtain the correct state $|\psi\rangle$. With probability at least $1-\epsilon$, this procedure succeeds with probability greater than $\frac{1}{1+\epsilon}$.

Let $U_{k}|\psi\rangle=\left(X_{k} \otimes I\right)\left|\Phi_{d}\right\rangle$. Note that $X_{k}$ is a $d \times n$ matrix where $n=(112 \ln 2)(d \log d) / \epsilon^{2}$. The entire protocol can be represented by the circuit

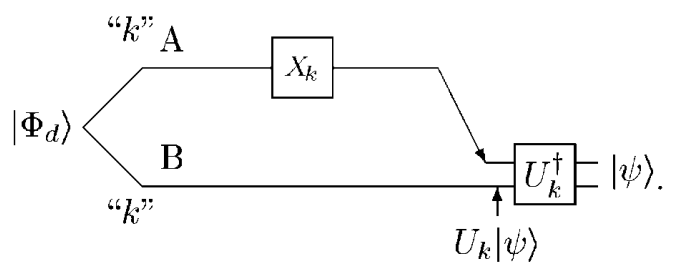

This protocol requires sending system $A$ of dimension $\frac{112 \ln 2}{\epsilon^{2}} d \log d$ and consuming $\log d$ ebits. If the goal is to prepare an arbitrary $2 l$-qubit state, we must take $d=2^{l}$ and, upon taking logs, we find that the protocol requires $l+\log l+2 \log \frac{1}{\epsilon}+7=l+o(l)$ qubits of communication, $l$ ebits, and $\log n \approx l+\log l+3 \log \frac{1}{\epsilon}+7=l+o(l)$ shared random bits. Asymptotically, this gives a rate of 2 remote qubits prepared for every qubit communicated, ebit consumed, and random bit shared.

Superdense coding of entangled states.-Our protocol for preparing pure states can be easily adapted to enable Alice and Bob to share a state $|\psi\rangle$ starting from $\left|\Phi_{d}\right\rangle$. We have an analog of the probabilistic protocol in Eq. (3):

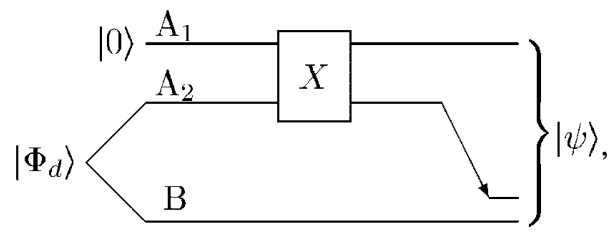

where again $|\psi\rangle=\left(X_{A_{1} A_{2}} \otimes I_{B}\right)\left|\Phi_{d}\right\rangle$. The nonunitary operation $X$ can be performed with probability $1 /\left(d \| \operatorname{Tr}_{B}|\psi\rangle\langle\psi| \|_{\infty}\right)$.

Again, the idea is to randomize $|\psi\rangle$ to make it highly entangled across the $A_{1} A_{2}$ vs $B$ partition. However, Bob has no access to $A_{1}$ so the randomization operation should only act on $A_{2}$ and $B$. The resulting protocol can be represented by the circuit

$$
\operatorname{Pr}_{U}\left(\operatorname{Tr}\left(\phi \operatorname{Tr}_{A} U \psi U^{\dagger}\right) \geq \frac{1}{d_{B}}+\frac{\epsilon}{2 d_{B}}\right)=\operatorname{Pr}_{U}\left(\operatorname{Tr}(I \otimes \phi)\left(U \psi U^{\dagger}\right) \geq \frac{1}{d_{B}}+\frac{\epsilon}{2 d_{B}}\right) \leq \exp \left(-\frac{d_{A} \epsilon^{2}}{14 \ln 2}\right),
$$

where the inequality follows from an argument almost identical to the proof of Lemma II.3 in [12].

Our second step is to prove that

$$
\operatorname{Pr}_{U}\left(\left\|\operatorname{Tr}_{A} U \psi U^{\dagger}\right\|_{\infty} \geq \frac{1}{d_{B}}+\frac{3 \epsilon}{4 d_{B}}\right) \leq\left(\frac{10 d_{B}}{\epsilon}\right)^{2 d_{B}} \exp \left(-\frac{d_{A} \epsilon^{2}}{14 \ln 2}\right) .
$$


$\mathcal{M}$ is called a $\delta$-net for pure states in an $m$-dimensional Hilbert space $\mathcal{H}$ if, for any pure state $\eta \in \mathcal{H}, \exists \tilde{\eta} \in$ $\mathcal{M}$ such that $\|\eta-\tilde{\eta}\|_{1} \leq \delta$. Here, $\|\cdot\|_{1}$ denotes the trace norm, which is the sum of the absolute values of the eigenvalues. Lemma II.4 in [12] states that $\exists \mathcal{M}$ such that $|\mathcal{M}| \leq\left(\frac{5}{\delta}\right)^{2 m}$. We always refer to this type of net in our discussion. Note that for any operator $O \in[0, I]$, $\operatorname{Tr}[(\eta-\tilde{\eta}) O] \leq \frac{\delta}{2}$. We will use this fact often, and we call it "fact $1 . "$

$$
\operatorname{Pr}_{U}\left(\left\|\operatorname{Tr}_{A} U \psi U^{\dagger}\right\|_{\infty} \geq \frac{1}{d_{B}}+\frac{3 \epsilon}{4 d_{B}}\right) \leq \operatorname{Pr}_{U}\left(\sup _{\tilde{\phi} \in \mathcal{M}_{B}} \operatorname{Tr}\left(\tilde{\phi} \operatorname{Tr}_{A} U \psi U^{\dagger}\right) \geq \frac{1}{d_{B}}+\frac{\epsilon}{2 d_{B}}\right) \leq\left(\frac{10 d_{B}}{\epsilon}\right)^{2 d_{B}} \exp \left(\frac{d_{A} \epsilon^{2}}{14 \ln 2}\right)=: \mu,
$$

where the last line is obtained using the union bound.

In our third step, we introduce the binary random variables

$$
X_{k}= \begin{cases}1 & \text { if }\left\|\operatorname{Tr}_{A} U_{k} \psi U_{k}^{\dagger}\right\|_{\infty} \geq \frac{1}{d_{B}}\left(1+\frac{3 \epsilon}{4}\right) \\ 0 & \text { otherwise }\end{cases}
$$

Then the $X_{k}$ are i.i.d. with expectation (over $U_{k}$ ) $\mathbb{E} X_{k} \leq \mu$ because of Eq. (9). For a fixed $\psi$,

$$
\operatorname{Pr}_{\left\{U_{k}\right\}}\left[\frac{1}{n} \sum_{k=1}^{n} X_{k}>\epsilon\right] \leq \exp [-n D(\epsilon \| \mu)] \leq \exp \left\{-n\left[\epsilon\left(-2 d_{B} \log \frac{10 d_{B}}{\epsilon}+\frac{d_{A} \epsilon^{2}}{14 \ln 2}\right)-1\right]\right\},
$$

where $D$ is the divergence, with

$$
D(\epsilon \| \mu):=\epsilon \log \epsilon+(1-\epsilon) \log (1-\epsilon)-\epsilon \log \mu-(1-\epsilon) \log (1-\mu) \geq-1-\epsilon \log \mu .
$$

Consider an $\frac{\epsilon}{2 d_{B}}$-net $\mathcal{M}_{A B}$ for $|\psi\rangle \in \mathcal{H}_{A} \otimes \mathcal{H}_{B}$. Then

$$
\operatorname{Pr}_{\left\{U_{k}\right\}}\left(\sup _{\tilde{\psi} \in \mathcal{M}_{A B}} \frac{1}{n} \sum_{k=1}^{n} X_{K}>\epsilon\right) \leq\left(\frac{10 d_{B}}{\epsilon}\right)^{2 d_{A} d_{B}} \exp \left\{-n\left[\epsilon\left(-2 d_{B} \log \frac{10 d_{B}}{\epsilon}+\frac{d_{A} \epsilon^{2}}{14 \ln 2}\right)-1\right]\right\},
$$

by the union bound. If

$$
n>\frac{2 d_{A} d_{B} \log \left(10 d_{B} / \epsilon\right)}{\left(\epsilon^{3} d_{A} / 14 \ln 2\right)-2 \epsilon d_{B} \log \left(10 d_{B} / \epsilon\right)-1},
$$

the probability in Eq. (12) is strictly less than 1 and there exists a choice of $\left\{U_{k}\right\}$ such that the corresponding event does not happen. That is,

$$
\sup _{\tilde{\psi} \in \mathcal{M}_{A B}} \frac{1}{n} \sum_{k=1}^{n} X_{K} \leq \epsilon
$$

Rephrasing the above using Eq. (10),

$\forall \tilde{\psi} \in \mathcal{M}_{A B}, \quad \operatorname{Pr}_{k}\left[\left\|\operatorname{Tr}_{A} U_{k} \tilde{\psi} U_{k}^{\dagger}\right\|_{\infty} \geq \frac{1}{d_{B}}\left(1+\frac{3 \epsilon}{4}\right)\right] \leq \epsilon$.

Finally, applying fact 1 to the net $\mathcal{M}_{A B}$,

$\forall \psi \in \mathcal{H}_{A B}, \quad \exists \tilde{\psi}$ such that

$$
\left\|\operatorname{Tr}_{A} U_{k} \psi U_{k}^{\dagger}\right\|_{\infty} \leq\left\|\operatorname{Tr}_{A} U_{k} \tilde{\psi} U_{k}^{\dagger}\right\|_{\infty}+\frac{\epsilon}{4 d_{B}} .
$$

Putting the last two equations together,

$$
\forall \psi \in \mathcal{H}_{A B}, \quad \operatorname{Pr}_{k}\left[\left\|\operatorname{Tr}_{A} U_{k} \psi U_{k}^{\dagger}\right\|_{\infty} \geq \frac{1}{d_{B}}(1+\epsilon)\right] \leq \epsilon .
$$

This completes the proof of Lemma 1 when we choose $d_{B}=d$ and $d_{A}=\frac{112 \ln 2}{\epsilon^{2}} d \log d$. With these parameters, and with the hypothesis $d_{B} \geq \frac{10}{\epsilon}$, it is straightforward to verify that $\frac{120 \ln 2}{\epsilon^{3}} d \log d$ is an upper bound to the required number of unitaries in Eq. (13).

[1] C. H. Bennett and S. J. Wiesner, Phys. Rev. Lett. 69, 2881 (1992).

[2] A. S. Holevo, Probl. Inf. Transm. 9, 177 (1973).

[3] D. Gottesman and I. L. Chuang, quant-ph/0105032.

[4] H.-K. Lo, Phys. Rev. A 62, 012313 (2000).

[5] C. H. Bennett, G. Brassard, C. Crépeau, R. Jozsa, A. Peres, and W. Wootters, Phys. Rev. Lett. 71, 1895 (1993).

[6] C. H. Bennett, P. Hayden, D.W. Leung, P.W. Shor, and A. Winter, quant-ph/0307100.

[7] A. Peres, Quantum Theory: Concepts and Methods (Kluwer, Dordrecht, 1995).

[8] S. Lloyd and H. Pagels, Ann. Phys. (N.Y.) 188 186, 1988.

[9] E. Lubkin, J. Math. Phys. (N.Y.) 19, 1028 (1978).

[10] D. Page, Phys. Rev. Lett. 71, 1291 (1993).

[11] A. Harrow, P. Hayden, and D.W. Leung, quant-ph/ 0307221.

[12] P. Hayden, D.W. Leung, P.W. Shor, and A. Winter, quant$\mathrm{ph} / 0307104$.

[13] A. Harrow, Phys. Rev. Lett. 92, 097902 (2004).

[14] J. J. Duistermaat and J. A. C. Polk, Lie Groups (SpringerVerlag, Berlin, 1999). 